\title{
CYP2D6 activity and the risk of recurrence of Plasmodium vivax malaria in the Brazilian Amazon: a prospective cohort study
}

\author{
Larissa W. Brasil ${ }^{1,2}$, Fernanda Rodrigues-Soares², Ana B. Santoro ${ }^{3}$, Anne C. G. Almeida ${ }^{1,2}$, Andrea Kühn', \\ Rajendranath Ramasawmy ${ }^{1,2,4}$, Marcus V. G. Lacerda ${ }^{1,2,5}$, Wuelton M. Monteiro ${ }^{1,2,4}$ and Guilherme Suarez-Kurtz ${ }^{3^{*}}$
}

\begin{abstract}
Background: CYP2D6 pathway mediates the activation of primaquine into active metabolite(s) in hepatocytes. CYP2D6 is highly polymorphic, encoding CYP2D6 isoforms with normal, reduced, null or increased activity. It is hypothesized that Plasmodium vivax malaria patients with defective CYP2D6 function would be at increased risk for primaquine failure to prevent recurrence. The aim of this study was to investigate the association of CYP2D6 polymorphisms and inferred CYP2D6 phenotypes with malaria recurrence in patients from the Western Brazilian Amazon, following chloroquine/primaquine combined therapy.
\end{abstract}

Methods: The prospective cohort consisted of P. vivax malaria patients who were followed for 6 months after completion of the chloroquine/primaquine therapy. Recurrence was defined as one or more malaria episodes, 28-180 days after the initial episode. Genotyping for nine CYP2D6 SNPs and copy number variation was performed using TaqMan assays in a Fast 7500 Real-Time System. CYP2D6 star alleles (haplotypes), diplotypes and CYP2D6 phenotypes were inferred, and the activity score system was used to define the functionality of the CYP2D6 diplotypes. CYP2D6 activity scores (AS) were dichotomized at $\leq 1$ (gPM, gIM and gNM-S phenotypes) and $\geq 1.5$ (gNM-F and gUM phenotypes).

Results: Genotyping was successfully performed in 190 patients (44 with recurrence and 146 without recurrences). Recurrence incidence was higher in individuals presenting reduced activity CYP2D6 phenotypes (adjusted relative risk $=1.89,95 \% \mathrm{Cl} 1.01-3.70 ; p=0.049$ ). Attributable risk and population attributable fraction were 11.5 and 9.9\%, respectively. The time elapsed from the first $P$. vivax malaria episode until the recurrence did not differ between patients with $\mathrm{AS}$ of $\leq 1$ versus $\geq 1.5(p=0.917)$.

Conclusions: The results suggest that CYP2D6 polymorphisms are associated with increased risk of recurrence of vivax malaria, following chloroquine-primaquine combined therapy. This association is interpreted as the result of reduced conversion of primaquine into its active metabolites in patients with reduced CYP2D6 enzymatic activity.

Keywords: Plasmodium vivax, Malaria recurrence, CYP2D6 gene, Polymorphisms, CYP2D6 activity score, Primaquine, Pharmacogenomics

\footnotetext{
*Correspondence: kurtz@inca.gov.br

${ }^{3}$ Coordenação de Pesquisa, Instituto Nacional de Câncer, Rio de Janeiro,

RJ 20231-050, Brazil

Full list of author information is available at the end of the article
} provided you give appropriate credit to the original author(s) and the source, provide a link to the Creative Commons license, and indicate if changes were made. The Creative Commons Public Domain Dedication waiver (http://creativecommons.org/ publicdomain/zero/1.0/) applies to the data made available in this article, unless otherwise stated. 


\section{Background}

Malaria is a global infectious disease, with 95 countries reporting transmission in 2015 and hundreds of millions of anti-malarial chemotherapies administered every year [1]. Anti-malarial drugs display considerable interindividual variability in disposition and clinical effects, whether beneficial or adverse, and there is evidence that genetic polymorphisms impacting drug pharmacokinetics and/or pharmacodynamics contribute to this variability [2-6]. Regarding Plasmodium vivax malaria, which prevails in the Brazilian Amazon where the present study was conducted, the World Health Organization (WHO) and the Brazilian Ministry of Health recommend combined chloroquine/primaquine as first-line therapy for uncomplicated disease in adults and children.

Primaquine is the only anti-malarial available clinically for prevention of $P$. vivax malaria relapses, which are classically associated with hypnozoite activation. From a pharmacogenomics perspective, the observation of primaquine-induced haemolysis in patients with G6PD deficiency [7] is not only a historical hallmark but a distinct example of clinical relevance of pharmacogenomicsinformed c deficiency [7]. G6PD deficiency prevalence was estimated in $4.5 \%$ in the Western Brazilian Amazon and was related to decreased malaria susceptibility [8], which led us to develop studies for the implementation of a rapid test to detect the G6PD deficiency in patients diagnosed with malaria. Comparatively, the impact of other pharmacogenomic polymorphisms in the response and/or disposition of primaquine and chloroquine in $P$. vivax malaria patients has drawn less attention [9]. However, an original observation by Bennet et al. [10] followed by a case report by Ingram et al. [11] triggered considerable interest in a novel aspect of the pharmacogenomics of $P$. vivax malaria. These reports described relapses of $P$. vivax following chloroquine/ primaquine combined therapy in three individuals with CYP2D6 genotypes encoding CYP2D6 isoforms with reduced (intermediate metabolizers) or null function (poor metabolizers). Relapses were ascribed to failure of primaquine hypnozoiticidal activity, which requires metabolic conversion of primaquine, a pro-drug, into redoxactive metabolite(s) [5].

Evidence from metabolomic studies in vitro [12] and animal models in vivo [13] indicate that the CYP2D6 pathway is largely responsible for primaquine activation in hepatocytes. Accordingly, P. vivax malaria patients with defective CYP2D6 function would be at increased risk for primaquine failure to prevent relapses [10, 11]. Indeed, St Jean et al. [14] verified a higher rate of relapse in intermediate CYP2D6 metabolizers, then in normal metabolizers in $P$. vivax patients from Peru and Thailand, and Silvino et al. [15] reported higher frequency of a CYP2D6 SNP (rs1065852) in multiple-relapse versus single-relapse patients from the Central-West region of Brazil.

The aim of this study was to investigate the impact of CYP2D6 polymorphisms and inferred CYP2D6 phenotypes on the risk of recurrence of $P$. vivax malaria in patients from the Brazilian Amazon, after completion of chloroquine/primaquine combined therapy.

\section{Methods \\ Study cohort}

A total of 213 patients living in a previously described community [16], located in the peri-urban area of Manaus, the capital city of the Amazonas state, were enrolled. All patients presented P. vivax malaria diagnosed by microscopy and confirmed by PCR [17] and were treated with the therapeutic scheme recommended by the Brazilian Ministry of Health for $P$. vivax uncomplicated malaria in children and adults, which consists of association of chloroquine $(150 \mathrm{mg} /$ day, 3 days $)$ and primaquine (15 mg/day, 7 day) [18]. Treatment was not supervised. Consanguineous individuals, pregnant women and children with less than 6 months of age were excluded from the study. The present analyses are based on data from 190 patients, who were successfully genotyped for CYP2D6 polymorphisms and copy number variation, and were followed up for 6 months after treatment, with monthly visits for blood sample collection and interviewing. P. vivax malaria recurrence was defined as one or more malaria episodes, 28-180 days after the initial episode.

\section{Laboratory procedures}

Genomic DNA was isolated from peripheral blood by standard procedures, using the QIAamp ${ }^{\circledR}$ DNA Mini Kit (QIAGEN $^{\circledR}$, Germany). CYP2D6 genotyping was performed as described previously [19], using a Fast 7500 Real-Time System (Applied Biosystems, Foster City, CA). TaqMan assays were used following the manufacturer's

\section{Table 1 CYP2D6 variants genotyped}

\begin{tabular}{ll}
\hline Variant & ID SNP \\
\hline$-1584 C>G$ & $\mathrm{rs} 1080985$ \\
$31 \mathrm{G}>\mathrm{A}$ & $\mathrm{rs} 769258$ \\
$100 \mathrm{C}>\mathrm{T}$ & $\mathrm{rs} 1065852$ \\
$1023 \mathrm{C}>\mathrm{T}$ & $\mathrm{rs} 28371706$ \\
$1846 \mathrm{G}>\mathrm{A}$ & $\mathrm{rs} 3892097$ \\
$2615-2617$ delAAG & $\mathrm{rs} 28371720$ \\
$2988 \mathrm{G}>\mathrm{A}$ & $\mathrm{rs} 28371725$ \\
$3183 \mathrm{G}>\mathrm{A}$ & $\mathrm{rs} 59421388$ \\
$4180 \mathrm{G}>\mathrm{C}$ & $\mathrm{rs} 1135840$ \\
\hline
\end{tabular}


protocols for allele discrimination at nine CYP2D6 polymorphic loci (Table 1) and for identification of CYP2D6 gene deletion and duplication/multiplication.

\section{CYP2D6 alleles and diplotypes}

CYP2D6 diplotypes were inferred using HaploStats software (version 1.7.7) implemented on the R platform. Software-generated haplotypes were compared to the Human Cytochrome P450 (CYP) Allele Nomenclature Database [20], for star (*) allele designation. The CYP2D6"1 allele was set when no nucleotide change was observed in all genotyped SNPs. Haplotypes not matched with known CYP2D6 alleles were grouped into the "other" category [19].

\section{Diplotype activity score and CYP2D6 phenotype}

The activity score (AS) system [21,22] was used to define the perceived functionality of the CYP2D6 diplotypes. Briefly, values of 0-2 were assigned to the alleles identified in the study cohort, as follows: zero, no-function alleles $(* 4, * 4 x N, * 5)$; 0.5 , decreased-function $(* 9, * 10, * 17, * 29$, *41); 1 , normal-function (*1, *2, *39) and 2, increasedfunction $\left({ }^{*} 1 x N,{ }^{*} 2 x N\right)$. The AS of diplotypes resulted from the sum of the assigned value to each allele. Patients with $\mathrm{AS}=0, \mathrm{AS}=0.5$, and $\mathrm{AS}>2$ were designated as genetic poor, intermediate, and ultrarapid metabolizers (gPM, gIM, and gUM), respectively. Patients with $\mathrm{AS}=1$, $\mathrm{AS}=1.5$, and $\mathrm{AS}=2$ were designated as genetic normal metabolizers (gNM), and sub-divided into gNM-slow (gNM-S, AS =1) and gNM-fast (gNM-F, AS = 1.5-2).

\section{Statistical analysis}

Allele and genotype frequencies of CYP2D6 polymorphisms were derived by gene counting. Deviations from Hardy-Weinberg equilibrium were assessed by the goodness-of-fit Chi square test. Chi square tests were also applied to compare frequencies of minor alleles at the nine polymorphic loci genotyped, star alleles, inferred phenotypes and activity scores. Data from Friedrich et al. [19] for healthy individuals from the Brazilian North region, which encompasses the Brazilian Amazon, were used for power calculations regarding CYP2D6 activity scores. A total of 45 patients with recurrence and 158 patients without recurrence confer a statistical power of 0.8 , at a one-sided $\alpha$ level of 0.05 to detect a relative risk of 2.5 for the combined frequency of activity scores ranging from 0 to 1 (gPM, gIM and gNM-S phenotypes, see above) versus $\geq 1.5$ (gNM-F and gUM). The relative risk (RR) of malaria recurrence among CYP2D6 phenotypes was assessed by univariate analysis, adjusting by age and sex using a logistic regression. A Hosmer-Lemeshow test was used to validate the models used. Attributable risk (AR) and population attributable fraction (PAF) were also calculated. A Kaplan-Meier survival analysis was performed in order to detect differences in the time elapsed from the baseline malaria episode to the first recurrence between patients with null (gPM) or reduced (gIM or gNM-S) CYP2D6 metabolic activity compared to gNM-F or gUM metabolizers. Log-rank test was used to test differences according CYP2D6 scores. Statistical significance was considered if $p<0.05$. Analysis was made as described in the Stata software manual.

\section{Results \\ Population characteristics}

Of the 213 individuals recruited to the study, 190 (44 with recurrence and 146 without recurrence) were successfully genotyped for CYP2D6 SNPs and copy number variation, and were included in the final analysis. Gender and age distribution were similar in both groups. Most of the patients presented only one recurrence episode (77.3\%). Recurrences were observed more frequently 60-180 days after the first malaria episode (68.2\%), with a mean time of 104 days ( \pm 40.7 ; CI 95\% 92.9-115.0) after the first episode. CYP2D6 activity score $\leq 1$ was $17.1 \%$ in the group with recurrence and $27.3 \%$ in the group without recurrence (Table 2).

\section{Distribution of CYP2D6 polymorphisms and activity scores} The minor allele frequency (MAF) at the nine CYP2D6 genotyped loci and the frequency distribution of the inferred CYP2D6 star alleles in the overall study cohort

Table 2 Demographic and clinical characteristics of the individuals included in the final analysis

\begin{tabular}{|c|c|c|c|}
\hline \multirow[t]{2}{*}{ Variable } & \multirow[t]{2}{*}{ Total (n, \%) } & \multicolumn{2}{|c|}{ Recurrence } \\
\hline & & No $(n, \%)$ & Yes (n, \%) \\
\hline Total & $190(100.0)$ & $146(76.8)$ & $44(23.2)$ \\
\hline \multicolumn{4}{|l|}{ Gender (\%) } \\
\hline Male & $107(56.3)$ & $84(57.5)$ & $23(52.3)$ \\
\hline \multicolumn{4}{|c|}{ Age (in years; \%) } \\
\hline $0-18$ & $81(42.6)$ & $62(42.5)$ & $19(43.2)$ \\
\hline $19-60$ & $98(51.6)$ & $75(51.4)$ & $23(52.3)$ \\
\hline$>60$ & $11(5.8)$ & $9(6.1)$ & $2(4.5)$ \\
\hline \multicolumn{4}{|c|}{ Malaria recurrence } \\
\hline 1 episode & $34(17.9)$ & - & $34(77.3)$ \\
\hline 2 episodes & $10(5.3)$ & - & $10(22.7)$ \\
\hline \multicolumn{4}{|c|}{ Time to first recurrence (in days) } \\
\hline $29-60$ & $14(7.4)$ & - & $14(31.8)$ \\
\hline $60-180$ & $30(15.8)$ & - & $30(68.2)$ \\
\hline \multicolumn{4}{|c|}{ CYP2D6 activity score } \\
\hline$\leq 1^{a}$ & $37(19.5)$ & $25(17.1)$ & $12(27.3)$ \\
\hline$\geq 1.5^{b}$ & $153(80.5)$ & $121(82.9)$ & $32(72.7)$ \\
\hline
\end{tabular}


are presented in Tables 3 and 4, respectively, along with data for healthy individuals from the Brazilian North region $[19,23]$.

Comparison of the two data sets reveals relatively small $(<5 \%$ of the total allele count), albeit significant differences $(p<0.05$, Chi square test) in MAF at the $31 \mathrm{G}>\mathrm{A}, 1023 \mathrm{C}>\mathrm{T}, 1846 \mathrm{C}>\mathrm{T}, 4180 \mathrm{G}>\mathrm{C}$ and $100 \mathrm{C}>\mathrm{T}$ SNPs (Table 3). Table 3 shows also the MAF at the nine CYP2D6 genotyped loci in patients with and without recurrence. Statistically significant deviations from Hardy-Weinberg expectations were not observed at any locus. The variant - 1584G allele (rs1080895) showed lower frequency in patients with recurrence $(p=0.015)$. No other significant differences in MAF were observed.

The distribution of the CYP2D6 star alleles among $P$. vivax malaria patients and healthy volunteers differed significantly $(p<0.001)$, but the difference in frequency of individual alleles did not exceed $5 \%$ of the total allele count. Alleles *10 and *39 were more frequent, whereas *17 was less frequent in the P. vivax patients (Table 4). The frequency distribution of the CYP2D6 star alleles differed significantly between patients with and without recurrences $(p<0.001$; Table 4$)$. The largest difference was in CYP2D6*2, which is defined by the $-1584 \mathrm{G}$ allele and was considerably less frequent in patients with recurrence $(p=0.148)$ than in those without recurrences (0.241). Differences in frequency of other star alleles did not exceed $5 \%$ of the total allele count. Regarding AS categories, a non- significant trend ( $p=0.075)$ for difference in frequency distribution among patients with and without recurrence was detected.

\section{CYP2D6 activity score and $P$. vivax malaria recurrence}

For this analysis, CYP2D6 activity scores (AS) were dichotomized at $\leq 1$, comprising gPM, gIM and gNM-S phenotypes, versus $\geq 1.5$, comprising gNM-F and gUM

Table 3 Minor allele frequency of CYP2D6 polymorphisms

\begin{tabular}{|c|c|c|c|c|}
\hline \multirow[t]{2}{*}{ Variant } & \multirow{2}{*}{$\begin{array}{l}\text { Friedrich } \\
\text { et al. [19] } \\
(n=474)\end{array}$} & \multicolumn{3}{|c|}{ Current study } \\
\hline & & $\begin{array}{l}\text { Total } \\
(n=380)\end{array}$ & $\begin{array}{l}\text { Recurrence } \\
(n=88)\end{array}$ & $\begin{array}{l}\text { No } \\
\text { recurrence } \\
(n=292)\end{array}$ \\
\hline$-1584 C>G$ & 0.229 & 0.203 & 0.115 & 0.229 \\
\hline $31 \mathrm{G}>\mathrm{A}$ & 0.024 & 0.005 & 0.00 & 0.006 \\
\hline $100 C>T$ & 0.142 & 0.197 & 0.214 & 0.192 \\
\hline $1023 C>T$ & 0.059 & 0.029 & 0.049 & 0.024 \\
\hline $1846 \mathrm{G}>\mathrm{A}$ & 0.128 & 0.086 & 0.088 & 0.085 \\
\hline 2615-2617delAAG & 0.010 & 0.009 & 0.000 & 0.012 \\
\hline $2988 G>A$ & 0.040 & 0.059 & 0.069 & 0.057 \\
\hline $3183 G>A$ & 0.024 & 0.011 & 0.000 & 0.015 \\
\hline $4180 G>C$ & 0.545 & 0.482 & 0.471 & 0.485 \\
\hline
\end{tabular}

$n$ number of chromosomes
Table 4 Frequency distribution of CYP2D6 star alleles

\begin{tabular}{|c|c|c|c|c|}
\hline \multirow[t]{2}{*}{ Variant } & \multirow{2}{*}{$\begin{array}{l}\text { Friedrich } \\
\text { et al. [19] } \\
(n=474)\end{array}$} & \multicolumn{3}{|c|}{ Current study } \\
\hline & & $\begin{array}{l}\text { Total } \\
(n=380)\end{array}$ & $\begin{array}{l}\text { Recurrence } \\
(\mathrm{n}=88)\end{array}$ & $\begin{array}{l}\text { No recurrence } \\
(n=292)\end{array}$ \\
\hline *1 & 0.418 & 0.409 & 0.409 & 0.456 \\
\hline *2 & 0.253 & 0.220 & 0.148 & 0.241 \\
\hline *4 & 0.112 & 0.086 & 0.102 & 0.082 \\
\hline$* 5$ & 0.027 & 0.024 & 0.068 & 0.010 \\
\hline *9 & 0.011 & 0.008 & 0.00 & 0.010 \\
\hline$* 10$ & 0.015 & 0.050 & 0.045 & 0.051 \\
\hline$* 17$ & 0.055 & 0.018 & 0.034 & 0.014 \\
\hline *29 & 0.023 & 0.013 & 0.000 & 0.017 \\
\hline *35 & 0.023 & 0.00 & 0.000 & 0.000 \\
\hline *39 & 0.015 & 0.060 & 0.102 & 0.048 \\
\hline$* 41$ & 0.042 & 0.047 & 0.068 & 0.041 \\
\hline Other & 0.000 & 0.024 & 0.023 & 0.024 \\
\hline \multicolumn{5}{|c|}{ Activity score (phenotype) ${ }^{b}$} \\
\hline 0 & - & - & 0.023 & 0.027 \\
\hline 0.5 & - & - & 0.023 & 0.027 \\
\hline 1 & - & - & 0.295 & 0.151 \\
\hline 1.5 & - & - & 0.182 & 0.137 \\
\hline 2 & - & - & 0.341 & 0.575 \\
\hline$>2$ & - & - & 0.136 & 0.082 \\
\hline
\end{tabular}

$n$ number of chromosomes

phenotypes. Incidence of $P$. vivax recurrences was $32.4 \%$ in patients with $\mathrm{AS} \leq 1$ and $20.9 \%$ in patients with $\mathrm{AS} \geq 1.5$ (crude $\mathrm{RR}=1.55$, CI 95\% 0.89-2.71; $p=0.075$ ). After adjusting for age and sex, a significant difference was noted between groups, with higher incidence of recurrence among patients with $\mathrm{AS} \leq 1.5$, i.e. presenting phenotypes of reduced CYP2D6 activity (adjusted $\mathrm{RR}=1.89$, CI 95\% 1.01-3.70; $p=0.049)$. AR and PAF were 11.5 and $9.9 \%$, respectively.

The time elapsed from the first $P$. vivax malaria episode until the recurrence did not differ significantly between the two groups of patients with AS $\leq 1$ or AS $\geq 1.5$ $(p=0.917$; Fig. 1$)$.

\section{Discussion}

The results of the present study point to increased risk of $P$. vivax malaria recurrence in chloroquine/primaquinetreated patients with null (gPM) or reduced (gIM or gNM-S) CYP2D6 metabolic activity compared to gNM-F or gUM metabolizers. This result is consistent with the requirement of CYP2D6-dependent conversion of primaquine into redox-active metabolites for hypnozoitical effect and with the original findings and conclusions in the case reports by Bennet and Ingram et al. [10,11]. The present study shows that CYP2D6 polymorphisms associate with $P$. vivax recurrence even in a scenario of active 
transmission, in which reinfections and poor adherence to primaquine regimens are possible [24]. Although recurrences have been associated mostly to subtherapeutic primaquine doses $[25,26]$, findings presented here show that strategies to achieve radical cure may also be hampered by the presence of poor or intermediate primaquine metabolizers in the population. New therapeutic regimens that do not require CYP2D6-mediated drug metabolism may reduce $P$. vivax recurrences in about $10 \%$ at the populational level in the field routine according to this finding. As a promising strategy, tafenoquine efficacy in $P$. vivax -infected individuals is not diminished to the same extent as primaquine in CYP2D6 intermediate metabolizers [14].

Higher relapse frequency in individuals categorized as intermediate metabolizes was also reported by St Jean et al. [14] but it is not clear whether their findings are comparable to this study, since their patients were apparently not genotyped for copy number variation, which is required for inference of CYP2D6 metabolic phenotypes. In another study of $P$. vivax relapse in Brazilians, Silvino et al. compared the frequency of SNPs at five CYP2D6 polymorphic loci between individuals with single or multiple-relapses. All individuals had a single CYP2D6 gene copy and the only significant difference between the two groups was higher frequency of the minor $100 \mathrm{~T}$ allele (rs1065852) among patients with multiple relapses [15]. This allele in combination with other SNP(s) define two CYP2D6 variants, namely the no function allele "4 and the reduced function allele *10. The study protocol could

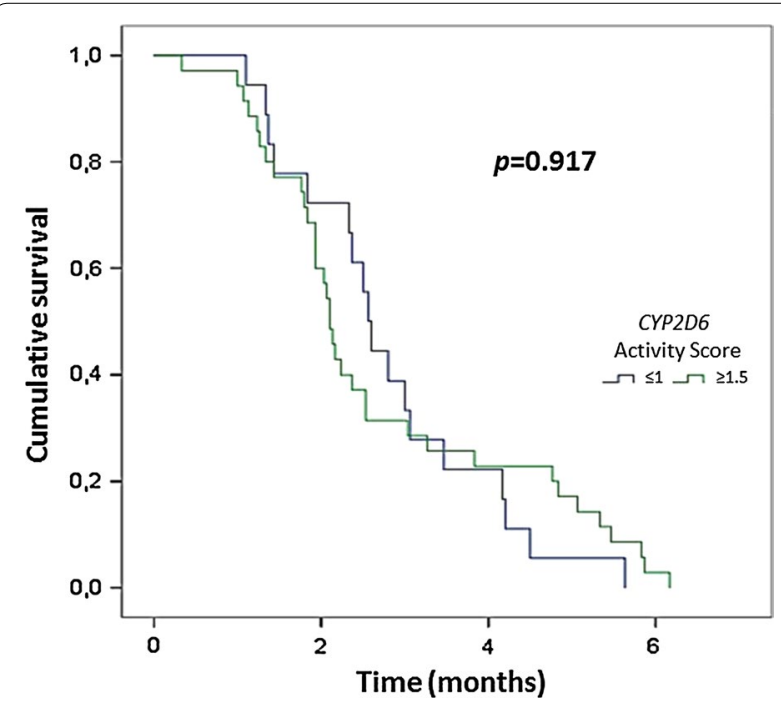

Fig. 1 Kaplan-Meier curve for malaria recurrence time between individuals with normal and reduced CYP2D6 activity phenotype. Score $\leq 1$ includes poor (gPM), intermediate (gIM) and normal-slow ( $\mathrm{gNM}-\mathrm{S}$ ) metabolizers and score $\geq 1.5$ includes ultrarapid (gUM) and normal-fast (gNM-F) metabolizers not distinguish these two alleles nor allowed for inference of the various CYP2D6 diplotypes known to occur in Brazilians [19].

This study has distinct strengths. First, it covered a considerably larger cohort of patients than previous reports of association of CYP2D6 polymorphisms and recurrence of $P$. vivax malaria. Second, inference of CYP2D6 phenotypes was based on genotype data from nine polymorphic loci and for gene copy number variation. Third, the frequency distribution of variants at the CYP2D6 polymorphic loci agreed well with data from healthy individuals from the Amazon region, where the subjects included in this study were recruited. Nevertheless, this study has limitations. Firstly, the prescribed anti-malarial treatment followed the recommendations of the Brazilian Ministry of Health for $P$. vivax, but primaquine administration was not supervised and the possibility of non-adherence to the 2 daily doses of primaquine during 7 days may not be excluded. Also, undisclosed use of non-prescribed drugs or xenobiotics that might affect CYP2D6 function cannot be excluded in this population. Thirdly, individual CYP2D6 phenotype was inferred from genotyping data, according to activity scores of CYP2D6 diplotypes and there is evidence for considerable range of variation in CYP2D6 function within genotype-inferred phenotype categories.

\section{Conclusion}

In a prospective cohort it was validated that polymorphisms in CYP2D6 gene associated with increased risk of recurrence of $P$. vivax malaria, following chloroquineprimaquine combined therapy in a field scenario. This association is interpreted as the result of reduced conversion of primaquine into its active metabolites in patients with reduced CYP2D6 enzymatic activity, leading to inappropriate hypnozoite elimination.

\footnotetext{
Abbreviations

AR: attributable risk; AS: activity score; CYP: cytochromes P450; CYP2D6: cytochrome P450 2D6; DNA: deoxyribonucleic acid; G6PD: glucose-6-phosphate dehydrogenase; gIM: intermediate metabolizer; gNM-F: normal fast metabolizer; gPM: poor metabolizer; gNM-S: normal slow metabolizer; gUM: ultrarapid metabolizer; MAF: minor allele frequency; PAF: population attributable fraction; PCR: polymerase chain reaction; RR: relative risk; SNP: single nucleotide polymorphism; WHO: World Health Organization.
}

\section{Authors' contributions}

LWB performed laboratory work and helped in paper drafted. ACGA and AK were responsible for the collection and organization of field samples. ABS helped in laboratory work and data analysis. FRS helped in the statistical analysis and manuscript revision and RR, MVGL, WMM and GSK participated in study design, coordination and elaborated the final version of manuscript. All authors read and approved the final manuscript.

\section{Author details}

${ }^{1}$ Diretoria de Ensino e Pesquisa, Fundação de Medicina Tropical Dr. Heitor Vieira Dourado, Manaus, AM, Brazil. ${ }^{2}$ Programa de Pós-Graduação em 
Medicina Tropical, Universidade do Estado do Amazonas, Manaus, AM, Brazil. ${ }^{3}$ Coordenação de Pesquisa, Instituto Nacional de Câncer, Rio de Janeiro, RJ 20231-050, Brazil. ${ }^{4}$ Faculdade de Medicina, Universidade Nilton Lins, Manaus, AM, Brazil. ${ }^{5}$ Instituto Leônidas \& Maria Deane, Fiocruz, Manaus, AM, Brazil.

\section{Acknowledgements}

We thank Sheila Vitor-Silva for her work with the field team. We acknowledge the collaboration of the local microscopists and the study subjects for their participation.

\section{Competing interests}

The authors declare that they have no competing interests.

\section{Availability of data and materials}

All data generated or analysed during this study are included in this published article and its can be made available files.

\section{Consent for publication}

All authors declare to consent of the manuscript and agree to the proposed authorship order.

\section{Ethics approval and consent to participate}

This study was approved by the Brazilian National Committee of Ethics (CONEP) (349.211/2013). All participants were informed about the objectives of the study as well as potential risks and benefits of their participation in the study. An informed consent form was signed by all study participants or by a parent or legal guardian in case of participants younger than 18 years. Children between 12 and 17 years signed an additional assent form.

\section{Funding}

This study was supported by Conselho Nacional de Desenvolvimento Científico e Tecnológico (CNPq) (Grant Numbers 575.788/2008-9, 442.442/2014-9 and 401.820/2014-9) and Fundação de Amparo à Pesquisa do Estado do Amazonas (Fapeam, Programa PAPAC Edital 015/2014). MVGL and GSK are level 1 research fellows from CNPq. RR is a research fellow from FAPEAM (PVS Program-PECTI-AM/PG\#019/2013). The funders had no role in study design, data collection and analysis, decision to publish, or preparation of the manuscript.

\section{Publisher's Note}

Springer Nature remains neutral with regard to jurisdictional claims in published maps and institutional affiliations.

Received: 17 May 2017 Accepted: 9 December 2017 Published online: 01 February 2018

\section{References}

1. WHO. World Malaria Report. Geneva: World Health Organization; 2016 http://www.who.int/malaria/publications/world-malaria-report-2016/ en/. Accessed 20 Oct 2017.

2. Gil JP. Malaria pharmacogenomics: return to the future. Pharmacogenomics. 2013;14:707-10.

3. Luzzatto L, Seneca E. G6PD deficiency: a classic example of pharmacogenetics with on-going clinical implications. Br J Haematol. 2014;164:469-80.

4. Dandara C, Swart M, Mpeta B, Wonkam A, Masimirembwa C. Cytochrome P450 pharmacogenetics in African populations: implications for public health. Expert Opin Drug Metab Toxicol. 2014;10:769-85.

5. Marcsisin SR, Reichard G, Pybus BS. Primaquine pharmacology in the context of CYP 2D6 pharmacogenomics: current state of the art. Pharmacol Ther. 2016;161:1-10.

6. Elewa $\mathrm{H}$, Wilby JJ. A review of pharmacogenetics of antimalarials and associated clinical implications. Eur J Drug Metab Pharmacokinet. 2017:42:745-56.
7. Dern RJ, Weinstein IM, Leroy GV, Talmage DW, Alving AS. The hemolytic effect of primaquine. I. The localization of the drug-induced hemolytic defect in primaquine-sensitive individuals. J Lab Clin Med. 1954;43:303-9.

8. Santana MS, Monteiro WM, Siqueira AM, Costa MF, Sampaio V, Lacerda MV, et al. Glucose-6-phosphate dehydrogenase deficient variants are associated with reduced susceptibility to malaria in the Brazilian Amazon. Trans R Soc Trop Med Hyg. 2013;107:301-6.

9. Sortica VA, Lindenau JD, Cunha MG, Ohnishi MD, Ventura AM, RibeiroDos-Santos AK, et al. The effect of SNPs in CYP450 in chloroquine/ primaquine Plasmodium vivax malaria treatment. Pharmacogenomics. 2016:17:1903-11.

10. Bennett JW, Pybus BS, Yadava A, Tosh D, Sousa JC, McCarthy WF, et al. Primaquine failure and cytochrome P-450 2D6 in Plasmodium vivax malaria. N Engl J Med. 2013;369:1381-2.

11. Ingram RJ, Crenna-Darusallam C, Soebianto S, Noviyanti R, Baird JK. The clinical and public health problem of relapse despite primaquine therapy: case review of repeated relapses of Plasmodium vivax acquired in Papua New Guinea. Malar J. 2014;13:488.

12. Pybus BS, Marcsisin SR, Jin X, Deye G, Sousa JC, Li Q, et al. The metabolism of primaquine to its active metabolite is dependent on CYP 2D6. Malar J. 2013;12:212.

13. Potter BM, Xie LH, Vuong C, Zhang J, Zhang P, Duan D, et al. Differential CYP 2D6 metabolism alters primaquine pharmacokinetics. Antimicrob Agents Chemother. 2015:59:2380-7.

14. St Jean PL, Xue Z, Carter N, Koh GC, Duparc S, Taylor M, et al. Tafenoquine treatment of Plasmodium vivax malaria: suggestive evidence that CYP2D6 reduced metabolism is not associated with relapse in the Phase $2 b$ DETECTIVE trial. Malar J. 2016;15:97.

15. Silvino AC, Costa GL, Araújo FC, Ascher DB, Pires DE, Fontes CJ, et al. Variation in human cytochrome P-450 drug-metabolism genes: a gateway to the understanding of Plasmodium vivax relapses. PLoS ONE. 2016;11:e0160172.

16. Brasil LW, Barbosa LR, Araujo FJ, Costa AG, Silva LD, Pinheiro SK, et al. TOLLIP gene variant is associated with Plasmodium vivax malaria in the Brazilian Amazon. Malar J. 2017;16:116.

17. Rosanas-Urgell A, Mueller D, Betuela I, Barnadas C, Iga J, Zimmerman PA et al. Comparison of diagnostic methods for the detection and quantification of the four sympatric Plasmodium species in field samples from Papua New Guinea. Malar J. 2010;9:361.

18. Brazilian Ministry of Health. Guia Prático de Tratamento da Malária no Brasil. Brasília: Brazilian Ministry of Health; 2010. p. 36.

19. Friedrich DC, Genro JP, Sortica VA, Suarez-Kurtz G, Moraes ME, Pena SD, et al. Distribution of CYP2D6 alleles and phenotypes in the Brazilian population. PLOS ONE. 2014;9:e110691.

20. Database THCPCAN. http://www.cypalleles.ki.se/cyp2d6.htm. Accessed 20 Jun 2017.

21. Gaedigk A, Simon SD, Pearce RE, Bradford LD, Kennedy MJ, Leeder JS. The CYP2D6 activity score: translating genotype information into a qualitative measure of phenotype. Clin Pharmacol Ther. 2008;83:234-42.

22. Gaedigk A, Sangkuhl K, Whirl-Carrillo M, Klein T, Leeder JS. Prediction of CYP2D6 phenotype from genotype across world populations. Genet Med. 2017;19:69-76.

23. Rede Nacional de Farmacogenética (Refargen). http://www.refargen.org. br/rubrique.php3?id_rubrique=36\&recalcul=oui. Accessed 20 Oct 2017.

24. Vítor-Silva S, Siqueira AM, Sampaio VS, Guinovart C, Reyes-Lecca RC, Melo GC, et al. Declining malaria transmission in rural Amazon: changing epidemiology and challenges to achieve elimination. Malar J. 2016;15:266.

25. Boulos M, Amato-Neto V, Dutra AP, Di Santi SM, Shiroma M. Análise da freqüência de recaídas de malária por Plasmodium vivax em região não endêmica. Rev Inst Med Trop Sao Paulo. 1991;33:143-6.

26. Duarte EC, Pang LW, Ribeiro LC, Fontes CJF. Association of subtherapeutic dosages of a standard drus regimen with failures in preventing relapses of vivax malaria. Am J Trop Med Hyg. 2001;65:471-6. 